\title{
Design of "Immersive" Teaching Situation Simulation System for Law Course Based on Artificial Intelligence
}

\author{
Yanyan Tian 10 \\ Shengda University of Economics, Business \& Management, Zhengzhou 451191, Henan, China \\ Correspondence should be addressed to Yanyan Tian; 100720@shengda.edu.cn
}

Received 9 January 2022; Revised 24 January 2022; Accepted 9 February 2022; Published 7 March 2022

Academic Editor: Baiyuan Ding

Copyright (c) 2022 Yanyan Tian. This is an open access article distributed under the Creative Commons Attribution License, which permits unrestricted use, distribution, and reproduction in any medium, provided the original work is properly cited.

\begin{abstract}
The method of scene mapping provides a very effective technical support for classroom participants to roam freely in a virtual scene larger than the real space. When the virtual scene is larger than the area in the real space, the existing scene mapping method will cause distortion of the original virtual scene and affect the user's roaming experience and visual experience. In order to obtain the scene map with low isometric distortion when the virtual scene scale is much larger than the real space, this paper proposes the block map to divide and group the roads in the virtual scene. For each group of roadblocks, the mapping is obtained by minimizing the isometric distortion energy of the mapping on the premise that the adjacent road blocks are smooth and continuous at the junction. The segmented roadblocks are mapped to the interior of the real intersections, and then the mapping of the whole scene is optimized globally to further reduce the isometric distortion of the whole mapping. Based on the above theories, the design scheme of immersive virtual legal reality classroom is proposed, including the overall framework of immersive virtual legal reality classroom, interactive scheme design, interactive behavior design, and required technical scheme, etc. The application technology, educational application, and application experience are analyzed in combination with the constructed immersive virtual legal reality scene for corresponding innovation and improvement. By applying immersive virtual reality technology in the field of education, the results show that the simulation system has good practicability and stronger stability and can provide a better immersive experience for law class participants.
\end{abstract}

\section{Introduction}

The 21st century was a period of transition from a primary society ruled by law to a higher society ruled by law. Modern law classroom teaching not only served law students' learning today but also laid a foundation for the sustainable development of law students tomorrow [1]. Among them, the evaluation of law classroom teaching was the foundation and key to the study and development of law students [2]. Modern law education paid attention to the role and value of law students in the future society, and the "high quality" talent education with innovative spirit and innovative ability required that law students must be guaranteed the subject status and ensure that law students were the leading role in learning activities [3].

The standard of law course points out that law teachers were the guiders of law study and law undergraduate students were the masters of law study [4]. Law undergraduate students were the masters of law learning activities, with independent subject consciousness and clear goals, and autonomy. They can actively accept the influence of legal education through a series of autonomous learning activities and self-regulation and transform legal knowledge into their spiritual wealth through legal practice [5]. The classroom was the main channel of legal education, the main link in the process of forming the knowledge of law students, and the main position for law teachers to complete the teaching task of legal education. With the development of science and technology, the traditional classroom teaching model had been unable to meet the needs of students to acquire knowledge, which to some extent limits the students' pioneering thinking, making their thinking only follow the development of teachers. The students' learning model was roughly the same, which limits their self-concept initiative 
[6]. Blended Learning combined the advantages of traditional learning with the advantages of E-learning [7]. However, most of the video resources of online learning were two-dimensional, and learners who were separated from the screen of the computer or mobile phone always study as a spectator, unable to fully integrate into the classroom behind the screen [8].

Education was changed by technology, and virtual reality was also applicable to education. As a technology made up for the defects, VR brought a new experience to education, cleverly avoiding the defects of two-dimensional video materials, changing the traditional two-dimensional into virtual three-dimensional, and giving students an immersive learning experience. Especially those who emphasized the spatial sense of education type, such as surgical medical station, sports competition tactical design, dance theater set coordination, a strong sense of interaction and impact of the law class; VR reproduces the teaching scene and accurately restores every detail to achieve an immersive experience. Nowadays, with the development of information technology, the problem of injecting technology into curriculum teaching and mobilizing students' subjective initiative could not be ignored. Motivation and behavior were not only the research emphasis of management but also was the key to the success or failure of education. In law classroom teaching, the student's learning motivation and learning investment was the key factor that influenced the learning result. Advanced information technology applications could promote the students' motivation; promoting students' learning investment behavior was the research focus on the reality of the problem. The full sensory and media richness of virtual reality technology broadens the dimension and deepens the level of technology-mediated perception. The influence of virtual reality teaching perception on teaching acceptance behavior, the effective mechanism, and the construction of the teaching model are the main contents of this study.

\section{Related Work}

The teaching context based on artificial intelligence, namely intelligent learning environment, had been expounded by domestic scholars in various fields from different perspectives. Zhong and Zhang [9] in 2007 defined an intelligent learning environment from the perspectives of constructivism learning theory, mixed learning theory, and modern teaching theory. They believed that intelligent learning environment was an intelligent, open, and integrated Virtual Reality (VR) learning space. Huang et al. [10] in 2012 put forward the system model and TRACE3 functional model of the intelligent learning environment and summarized the support of current technological development for the construction of intelligent learning environment from artificial intelligence, sensor technology, and communication technology. Nie et al. [11] 2013 constructed the ISMART model of the smart classroom from the perspective of system components, which consists of six systems including infrastructure, network awareness, visual management, augmented reality, real-time recording, and ubiquitous technology. Hwang [12] 2014, from the perspective of ubiquitous situational perception, believed that a smart learning environment was a technology-supported learning environment, which could be adjusted at an appropriate time and place according to learners' needs and provided appropriate support (such as guidance, feedback, and tools). Shi [13] 2017 explored the deep integration of information technology and smart classroom construction based on the web of things, cloud computing, VOIP, video surveillance, and other technologies and proposed the overall architecture diagram of a smart classroom system. Pan et al. [14] 2018 introduced speech processing technology into the construction of a smart classroom. Zero-intervention classroom data collection was achieved by mounting microphones, and acoustic wave recognition and speech recognition algorithms were designed to realize speaker identification and recording of the classroom discussion process, and the results were fed back to students and teachers in class in realtime. Zhang et al. [15] 2019 explored the mechanism of influencing factors of college students' learning engagement by using multiple regression methods from the perspectives of students' own factors, teachers and peers, and intelligent learning environment.

Foreign scholars had conducted in-depth research on intelligent learning environments. Kim et al. [16] in 2011 introduced the concept of Elastic Four Smarts (E4S) to build a smart learning cloud environment that could realize situational awareness, focused on predicting potential needs of learners and matched relevant learning content by collecting and analyzing behavioral data of learners, pushing it with the help of smart learning cloud environment, so as to provide smart learning services for learners. Singapore scholar $\mathrm{Wu}$ and Looi [17] 2012 emphasized the importance of learners' reflection in the intelligent learning environment, aimed to trigger students' double-cycle reflection on metacognitive strategies and beliefs. Specific prompts encourage students to reflect on a single cycle of related fields and task-specific skills. Malaysian scholar Cheng and Shan [18] 2013 believed that a smart learning environment should be learner-centered, with the ability to push learning content based on learners' knowledge base, learning style, and learning ability. It provided support for learners' lifelong learning and development. Information and communication technology was the foundation for building a smart learning environment. Thai scholar Temdee [19] pointed out in 2014 that the key to learning was to make learners in a learning environment with situational awareness function, and they would not even be aware of their own learning process. Based on a multiagent framework, a ubiquitous intelligent learning environment was developed which could adaptively push learning content in an appropriate way to promote learners' autonomous learning and collaborative learning. Spanish scholar Griol et al. [20] pointed out that artificial intelligence, immersive virtual environment, and natural language processing create more possibilities for the research and development of intelligent learning environments. They proposed a method to create an intelligent learning environment in Second Life, Open Simulator, and other games. Canadian scholar Kinshuk et al. [21] 2016 redesigned the basic structure and function of the current 
learning environment to better combine emerging technologies with the required teaching transformation so as to completely transform the current learning environment into a smart learning environment. German scholars Le and Pinkwart [22] 2019 proposed an intelligent learning environment model based on constructivism learning theory. That wisdom was mainly reflected in the following: First, it can provide learners with real problems existing in reality; Second, it could provide information retrieval or collection tools to help learners in the problem-solving process; Third, problem generation tools could facilitate learners' reflection and thinking processes.

To sum up: in this paper, combined with virtual reality and immersive virtual reality of the core concepts, characteristics, and research status at home and abroad, put forward the VR and technology application in law class of immersive virtual reality simulation system, including the overall framework of immersive virtual reality classroom, interactive design, interaction design, and technical solutions required. The immersive virtual reality classroom was constructed with the real simulation theory of large scenes based on block mapping. The educational application and application experience were analyzed through experiments, and continuous innovation and improvement were made. The application of immersive virtual reality technology in the field of education and the analysis of the educational application value of immersive virtual reality classrooms were bold attempts of modern artificial intelligence technology in the direction of education, which was bound to promote the development of educational technology and provide the theoretical basis for the realization of the modernization of education.

\section{Real Simulation Theory of Large Scene Based on Block Mapping}

In order to roam the large scene in the small real space, it is necessary to control the user in the specific real space at all times and make the user's visual perception and motion perception maintain the same consistency with real life. In order to meet these requirements, researchers have proposed a variety of methods in recent years $[50,128,153]$, such as redirecting walking technology, manipulating scene structure, and so on. The most widely used redirected walking technique involves forcing the user to interrupt his roaming and reset his direction of motion whenever he reaches the boundary of real space. Such operations greatly affect users' roaming experience. Recently, Sim [13] proposed a method based on scene mapping to realize real walking and roaming virtual scenes. The method calculates the smooth mapping from the virtual scene to the inner part of the real space so as to avoid the user touching the boundary of the real space in the process of walking and roaming and avoid the incoherent experience caused by forcibly interrupting the user's roaming process. However, this method is a global mapping method. When the scale gap between virtual scene and real space is large, the scene mapping generated by this method will cause great distortion of the scene, as shown in Figure 1. This will produce an unacceptable visual experience when the user is roaming and even lead to the failure of real walking and roaming. Computing scene maps with low distortion for large scenes remains challenging.

In order to map superlarge virtual scenes into small real Spaces with low isometric distortion, a new mapping method named block mapping is proposed in this paper. Different from the global mapping method proposed by Sim et al., this method is based on the divide-and-conquer strategy. It creates multiple fragmented local mappings for the virtual scene and maps each corresponding area to the interior of the real space. Firstly, the virtual scene is divided into small area blocks, and each area block is mapped to the interior of the real space by minimizing the mapping isometric distortion energy. In the process of optimizing local mapping, we need to keep smooth continuity between adjacent area blocks. After obtaining all local mappings, we can optimize the mapping of the whole virtual scene globally. In the process of global optimization, additional consideration should be given to the continuity of loop closure in the virtual scene and the obstacle avoidance requirement when the road in the whole scene is mapped to the interior of the real space. In addition, the block mapping algorithm can also map the specific areas of multiple virtual scenes to the same real location so that different virtual scenes can transfer and transform each other using transfer points. Compared with previous mapping methods, block mapping can generate high-quality scene mapping with low distortion for very large virtual scenes so as to provide a highly realistic immersive experience for virtual reality applications combined with real walking.

3.1. Block Mapping Algorithm. The algorithm input includes the plane plan of the virtual scene and plane plan of real space SV, and plane plan of real space $S^{R} . S^{V}>S^{R}$ and even $S^{V} \gg S^{R}$ in general. In practice, for a virtual scenario, only the area within which users can walk is considered, that is, the network within the $S^{V}$. For the sake of generality, the walkable area in the virtual space is taken as the actual input and is still denoted as $S^{V}$.

The problem of realizing real roaming using scene mapping can be abstracted as calculating a smooth mapping $f: S^{V}-S^{R}$ according to some conditions and constraints, which can map every point to point $(x, y) \in S^{R}$.

A global mapping from $S^{V}$ to $S^{R}$ may produce a large distortion. A divide-and-conquer strategy is proposed to calculate the mapping. $S^{V}$ is first decomposed into $\mathrm{K}$ region blocks such that. Each region block $P_{k}^{V}$ can be independently mapped to the interior of SR, resulting in a low distortion local map $f_{k}: P_{k}^{V} \longrightarrow S^{R}$. All local maps $\left\{f_{k}\right\}_{k=1}^{K}$ are assembled together smoothly to obtain the global map $f: S^{V} \longrightarrow S^{R}$.

There are two technical difficulties in stitching all local maps $\left\{f_{k}\right\}_{k=1}^{K}$ together to produce A smooth map $f: S^{V} \longrightarrow S^{R}$. First of all, it is very important to map large virtual scenes into small real Spaces and maintain low isometric distortion to provide a good experience for users to roam the virtual scenes, but this is a very difficult thing to do. In addition, the local mapping corresponding to two 


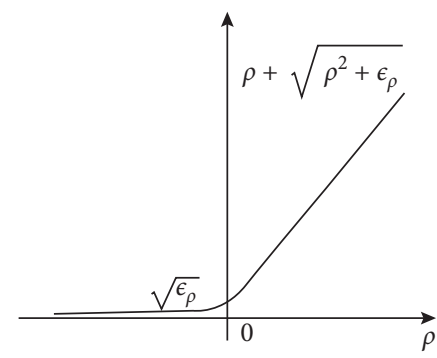

(a)

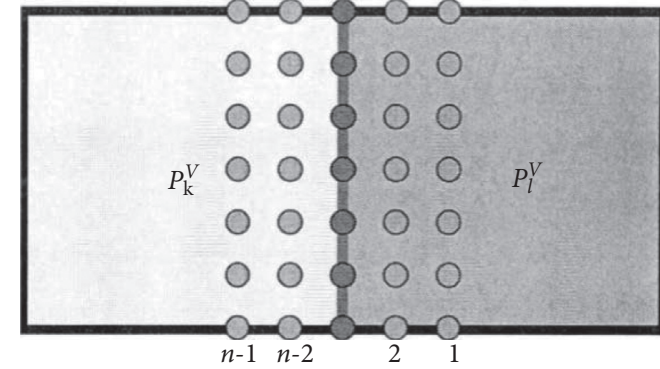

(b)

FIgURE 1: (a) Schematic diagram of the boundary cost function. (b) Smooth continuity diagram.

adjacent area blocks should be smooth enough at the junction; otherwise, users will experience unnatural jumps across area blocks in the process of roaming the virtual scene. Inspired by spline theory, local maps are expressed in the form of tensor product Bezier surface maps (assuming $P_{k}^{V}$ is a rectangular region). This representation is very convenient to maintain the smoothness of the adjacency during the assembly of the local map. It is necessary to ensure that the control points of the regional block maintain corresponding linear constraints during the assembly. Other constraints on the map can be easily implemented in this representation.

3.1.1. Mapping Represents. Let's call the region block $P_{k}^{V}=\left[a_{k}, b_{k}\right] \times\left[c_{k}, d_{k}\right]$. And express the mapping as $f_{k}: P_{k}^{V} \longrightarrow S^{R}$ in the form of a tensor product Bezier surface mapping:

$f_{k}(u, v)=\sum_{i=0}^{n} \sum_{j=0}^{m} c_{i, j}^{k} B_{i}^{n}\left(u^{*}\right) B_{j}^{m}\left(v^{*}\right),(u, v) \in P_{k}^{V}$,

where $\left\{B_{i}^{n}(\cdot)\right\}_{i=0}^{n}$ and $\left\{B_{j}^{m}(\cdot)\right\}_{j=0}^{m}$ are Bernstein polynomial bases of order $n$ and $m$, respectively. $u^{*}=u-a_{k} / b_{k}-a_{k}$, $v^{*}=u-c_{k} / d_{k}-c_{k}$ and $(n+1) \times(m+1)$ control points $\left\{c_{i, j}^{k}\right\}_{i=0, j=0}^{n, m}$ are the variables that define this mapping. To measure the quality of the mapping $f_{k}$. Uniformly sample $N_{k}$ sample points $Z_{k}=\left\{z_{k, l}\right\}_{l=1}^{N_{k}}$ in $P_{k}^{V}$ and calculate various cost functions at each sample point. After weighing the flexibility and size of the variables, $n=m=7$ and $N_{k}=3600$ are implemented.

3.1.2. Distortion Cost Function. Distortion cost using isometric energy measure $f_{k}\left(z_{k, l}\right)$.

$$
E_{K, l}^{\mathrm{dis}}=\sum_{j=1}^{2}\left[\left(\sigma_{K, l}^{j}\right)^{2}+\left(\sigma_{K, l}^{j}\right)^{-2}\right]=\left\|J_{K, l}\right\|_{F}^{2}+\frac{\left\|J_{K, l}\right\|_{F}^{2}}{\left(\operatorname{det} J_{K, l}\right)^{2}},
$$

where $J_{K, l}$ represents the Jacobian matrix of $f_{k}, z_{K, l}, \sigma_{K, l}^{1}, J_{K, l}$ is the singular value of $J_{K, l}$, and $\|\cdot\|_{F}$ represents the Frobenius norm $u$. If det $J_{K, l}>0$, namely $f_{k}$ local bijection, $E_{K, l}^{\text {dis }}$ reaches the minimum when all the singular values are $1 . E_{K, l}^{\text {dis }}$ approaches infinity as det $J_{K, l}$ approaches 0 to prevent $f_{k}$ from degenerating and flipping.
3.1.3. Boundary Cost Function. The directed distance that maps $f_{k}\left(P_{k}^{V}\right)$ completely into the $j$ boundary of $\mathrm{B}$, If $f_{k}\left(z_{k, l}\right) \in S^{R}, d_{k, l}^{j}>0, \varepsilon_{d}$ is a positive small quantity to compensate for the negative directed distance, when $d_{k, l}^{j}>0$, the denominator of this energy becomes extremely small, making $E_{K, l}^{\text {dis }}$ extremely large.

3.1.4. Total Cost Function. The above two cost functions are combined to measure the quality of the local mapping $f_{k}$ from $P_{k}^{V}$ to $S^{R}$.

$$
E\left(P_{k}^{V}, S^{R}\right)=\frac{1}{N_{k}} \sum_{l=1}^{N k}\left(\omega_{\mathrm{bnd}} E_{k, j}^{\mathrm{dis}}+\omega_{\mathrm{dis}} E_{k, j}^{\mathrm{bnd}}\right),
$$

where $\omega_{\text {dis }}$ and $\omega_{\text {bnd }}$ are the weights of the distortion cost function and the boundary cost function, respectively, and the default values are $\omega_{\text {dis }}=5$ and $\omega_{\text {bnd }}=1$.

3.1.5. Smooth Continuity Constraint. In order to ensure smooth continuity of the local mapping connection for two adjacent area blocks $P_{k}^{V}$ and $P_{l}^{V}$ with common edges, to ensure that the corresponding local mapping $f_{k}$ and $f_{t}$ have $C^{2}$ connectivity at the connection, the following constraints are applied to their control points:

$$
\begin{aligned}
c_{n, j}^{k} & =c_{0, j}^{l} \\
\frac{c_{n, j}^{k}-c_{n-1, j}^{k}}{b_{k}-a_{k}} & =\frac{c_{1, j}^{l}-c_{0, j}^{l}}{b_{l}-a_{j}} \\
\frac{c_{n, j}^{k}-2 c_{n-1, j}^{k}+c_{n-2, j}^{k}}{\left(b_{k}-a_{k}\right)^{2}} & =\frac{c_{2, j}^{l}-2 c_{1, j}^{l}+c_{0, j}^{l}}{\left(b_{l}-a_{l}\right)^{2}} .
\end{aligned}
$$

Among them $j=0,1, \ldots, m$. The above formula is linear for the control point $\left\{c_{i, j}^{k}\right\}_{i=0, j=0}^{n, m}$, so it can be expressed as follows:

$$
A C=b,
$$

where $C$ is a vector composed of control points.

The local mapping $f_{k}$ on the A region block $P_{k}^{V}$ is obtained, and the local mapping force $f_{1}$ on the adjacent region block $P_{l}^{V}$ can be obtained while minimizing the constraint $A C=b$. For each region block, the degree of freedom of the 
variable is not enough to satisfy the smooth continuity constraint and optimize the algorithm to obtain a map with low distortion energy. Therefore, we combine some adjacent area blocks together to form superpatches so that each superpatch can have enough degrees of freedom to obtain local mappings that meet the conditions through optimization. After the mapping calculation and assembly of all regional blocks are completed, we proceed to a global optimization to further reduce the mapping distortion:

$$
\left\{\begin{array}{l}
\min _{\left\{c_{i, j}^{k}\right.} \sum_{k=1}^{K} E\left(P_{k}^{V}, S^{R}\right), \\
\text { s.t. } A C=b .
\end{array}\right.
$$

Some additional constraints need to be considered during global optimization. First, if the $S^{V}$ contains a circular road, the constraint of smooth continuity between adjacent area blocks in the circular road needs to be added to the above equation. On the other hand, if there is an obstacle inside $S^{R}$, the mapping of $S^{V}$ needs to avoid overlapping with the obstacle. The cost function in $E\left(P_{k}^{V}, S^{R}\right)$ the above formula is modified, and the internal obstacle punishment function is added. The penalty function added for each obstacle is expressed as a two-dimensional Gauss-based barrier function.

\section{Construction Process of Immersion Law Classroom Situation}

The construction of immersive virtual reality classroom mainly uses 3ds Max software for basic model modeling and material endowing, and Pano2VR was used to transform the virtual classroom into panoramic mode. The virtual reality classroom constructed this time did not realize real interaction. After all, a completely immersive virtual reality environment requires a mature technology research and development team. Before establishing a virtual reality classroom, it should know what objects there were in the classroom, what the size was, and what the specific values of the length, width, and height of the classroom were, which was the preliminary preparation for a basic scene modeling. Reference to the real classroom virtual classroom modeling, so that students in a real teaching situation could reduce the distraction of attention, more concentrate on learning.

\subsection{Realization of Immersive Virtual Reality Classroom} Modeling. Immersive virtual reality classroom is mainly composed of two parts. One is to create a virtual reality classroom environment using 3DS MAX modeling software and Unity $3 \mathrm{D}$ scene integration software. The second is the interaction with the virtual reality classroom environment. Teachers and students need to wear the helmet of virtual reality, auxiliary equipment necessary sensor immersed in the virtual classroom environment, the virtual reality system based on the teachers' and students' body displacement, gestures, eye location and tracking head, voice recognition, such as signal input is explained, and then to the virtual reality environment database updates, real-time adjustment of the virtual environment; Then the 3D scene information from the new viewpoint is immediately transmitted to the head-mounted display and other corresponding sensory experience.

Immersive virtual reality classrooms had tables, chairs, lecturers, blackboards, doors and windows, floors, and wall charts for advertising, just like traditional classrooms. The area of the classroom, the length, width, and height of the desks and chairs were modeled according to the standard size. Construct a classroom that could accommodate 30 people, 9 meters long, 6.6 meters wide, and 3 meters high. There were many ways to model, of course, the first choice was to use the standard basic body for modeling, cuboids, cylinders, such as walls, table legs, doors, window frames; Secondly, the angular cuboid and angular cylinder in the extended basic body were selected for modeling, such as desktop, chair, blackboard, wall chart frame, etc. Furthermore, the line, rectangle, circle, polygon in the spline line were selected, and the modeling was carried out by creating the lofting method in the composite object, such as the circular arc rod of the table and chair, curtain, etc. Finally, some more complex models, such as Windows and doors, could be completed by using two simple standard cuboids and Boolean operations. For the complex model that was difficult to construct, the object could be modeled by displaying the background image in the viewport background, tracing the two-dimensional basic shape of the object, and performing the "extrude" operation command in the editor or modifier.

The modeling of desks in the virtual classroom was completed in $3 \mathrm{~d}$ s Max software. Before modeling the object, be sure to set the units. In the custom menu bar, set the system units to centimeters. Select the cuboid in the extended basic body of the command panel, set the parameters as length: $60 \mathrm{~cm}$, width: $40 \mathrm{~cm}$, height: $2 \mathrm{~cm}$, and rounded corner: $50 \mathrm{~cm}$. Drag the mouse in the top view to complete the modeling of the desk surface. Select the cuboid again and set the length as $38 \mathrm{~cm} ; 36 \mathrm{~cm}$ wide, $17 \mathrm{~cm}$ high, $5 \mathrm{~cm}$ rounded, and converted to editable polygons. In the Edit modifier, select the polygon command, then select a side face to delete, add a "shell" to the object in the modifier list to increase the thickness, and the drawer modeling was complete. The circular pole on the side of the table is constructed by lofting. In the spline, select a line, draw a smooth curve in the left view as the lofting path, and adjust the radian and position of the curve; Then draw a cuboid with a length of $52 \mathrm{~cm}$, a width of $2.5 \mathrm{~cm}$, and a rounded angle of $1 \mathrm{~cm}$ as the lofting figure. Finally, click the curve to create a composite object, select Lofting, and select Get Graph in the creation method. The other legs of the desk are also made of cuboids in the standard basic body. Select all the parts of the desk, select groups from the menu bar, and then "group" operation, named Desk, and make them into a whole. In order to improve the computing efficiency of the computer, only the Max file with one desk was saved, and finally, when combined together, 30 desks were copied by the "array" method. The finished desk model is shown in Figure 2. 


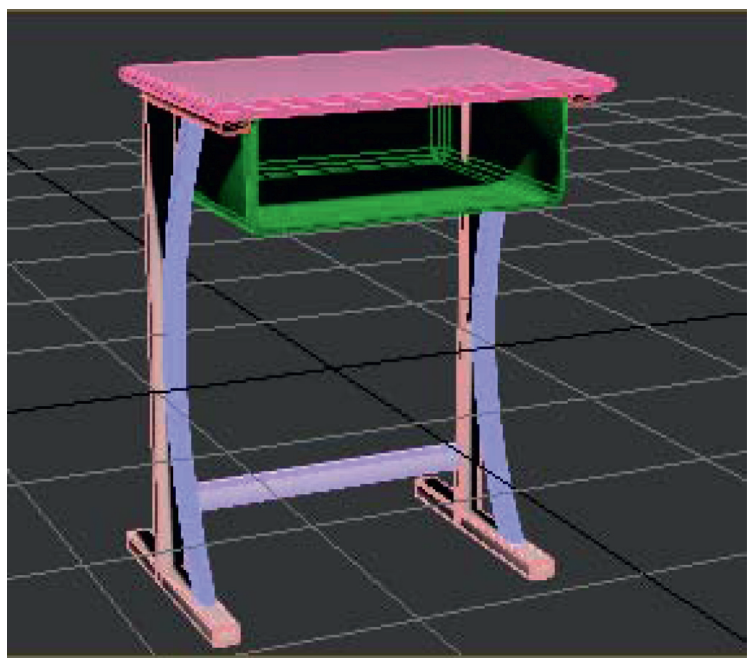

Figure 2: Classroom desk modeling diagram.

4.2. Material Endowing of Immersive Virtual Reality Classroom. After the basic modeling was completed, it also needed to assign materials to them, select the compact material mode, adjust the texture ball, bump, reflection, refraction, highlight, and other properties, and then assign the texture ball to the objects in the scene to complete the texture mapping operation. For some complex objects, you could choose to use UVW expansion or multidimensional subobjects to texture the object.

First, open the material editor and select the desired bitmap "desktop" in diffuse. JPG “, set the highlight level to 15 and glossiness to 20 in the reflection highlight. The material ball was then assigned to the tabletop in the scene to complete the material mapping of the tabletop. Select another material ball, set the highlight level to 35, glossiness to 10 , and diffuse color to white, and assign it to the desk drawers, desk legs, etc. The final rendering of the desk material diagram is shown in Figure 3.

For classroom windows, doors, and other objects with glass, to give them material, but also on its transparency settings. Glass was also modeled using simple cuboids. In other words, glass was also a solid object, but it was transparent. Select a blank material ball in the Material editor, lower the opacity, and assign it to the window.

\subsection{Lighting Setting of Immersive Virtual Reality Classroom.} Once the material was finished, the next step was to lay out the lights for the scene. The scene had default lighting, but the default lighting could only be single rendered. So to make the virtual reality classroom look more realistic, "photometric" lighting was used to simulate sunlight in real life. Photometric lighting was relatively simple, and you could choose the appropriate bulb lighting template, exposure control parameters, lighting color, atmospheric effect, attenuation range, and other parameters that could be set to achieve the simulation of the real classroom light. Using photometric lighting for precise numerical Settings could make the scene more realistic. In the Create panel, select Photometry, click Target Light in the object type, and drag in

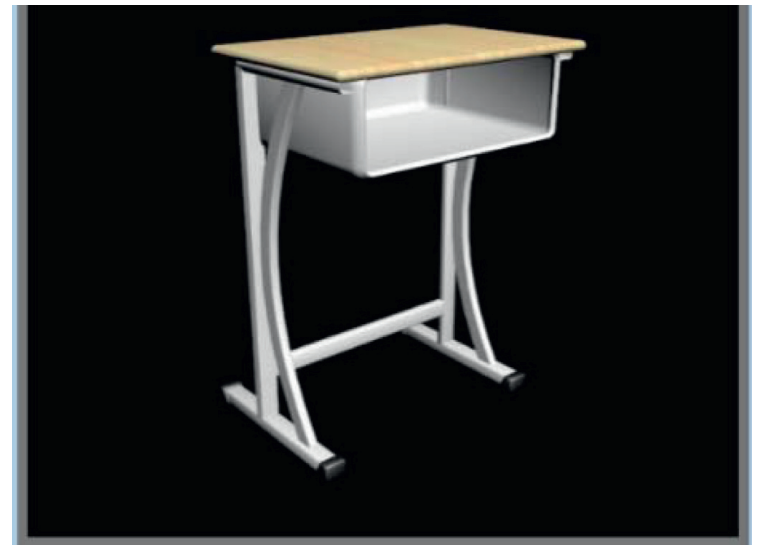

FIGURE 3: Classroom desk material rendering.

the left view to finish creating the light. In the modify Settings bar, select Enable Shadow, set the creation parameters, select Exposure control in the "Render" menu, set the brightness, contrast, and other values of exposure control parameters in the dialog box that appears, add volume light to make the scene had the smoke effect of light. Create two more suitable free lights in the classroom and adjust the position of the lights in the view to complete the lighting of the scene. Free lighting was set up similarly. After modeling, material, and lighting operations, you could set up a camera in the scene, choose the appropriate position, set up a good perspective for the scene, easy to choose the perspective for observation. Figure 4 shows the virtual classroom rendered after lighting Settings.

\subsection{Interactive Technology of Immersive Virtual Reality} Classroom. The immersive interactive experience of virtual reality classrooms mainly comes from the realistic THREEDIMENSIONAL display and accurate positioning and tracking so that teachers and students can have a feeling of being in the classroom. The key to interact with an immersive VR classroom was location tracking technology 


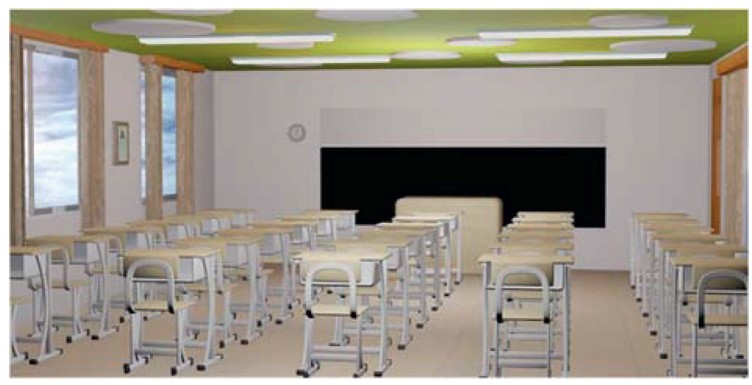

FIgURE 4: Virtual classroom rendering after lighting setting.

such as head tracking, motion tracking, eye movement tracking, and recognition technology such as speech recognition and gesture recognition.

In order to simulate the natural visual transformation effect of human, the head-mounted display was usually equipped with a head tracking function. In terms of motion tracking, supporting controllers were required, such as Oculus Rift's Touch controller, SONY Playstation's PS Camera and Move controller, HTC Vive's laser sensor controller, etc. A large number of sensors were built into the controller. In order to achieve the movement effect of the scene. Supporting SDKS was also an essential resource for implementing interactive behavior. In immersive virtual reality classrooms, teachers and students control their vision through head movements and act as a mouse by capturing the fixation point or movement trajectory of the eyes to become spontaneous and more in line with the feeling of real life. FOVE was an eye-tracking device that used infrared tracking sensors built into a head-mounted display to track eye movements and enhance the sense of presence.

Leap Motion, Kinect, and other depth sensors were used to realize gesture recognition. These sensors could accurately detect the position and movement of the hand, obtain depth image information, and perform calculations to obtain data on the bending angle of the hand, finger, and so on. In terms of speech recognition, it was very difficult for a virtual reality environment to understand human language and interact with people in real time.

\subsection{Interactive Realization of Immersive Virtual Reality} Classroom. Due to the lack of necessary head-display equipment and other auxiliary equipment in the construction process of the virtual reality classroom, the real immersive virtual reality experience could not be realized. The virtual reality classroom could only generate web pages through Pano2VR software to complete the 360-degree panoramic roaming experience. In Pano2VR, hot area interaction was completed by adding hot spot interaction. Clicked the hot interactive button in the virtual classroom and entered the virtual classroom for law study by linking online resources. First, import a panorama image into Pano2VR, select add noninteractive hotspot and move the interactive hotspot button to the appropriate position. At this time, the hotspot button was red, indicating that no Settings had been carried out. Set the ID to Point01, type to URL, skin ID to the hotspot, title to Video, and you could add the corresponding main content description. The most important thing was to set the URL and destination address of the link target. Enter the target address of the online resource you wanted to link to here so that you can accurately open the externally linked resource when browsing the web page. The set hot button would turn blue. Students enter the virtual classroom from the master perspective of "firstperson", immerse themselves in the classroom from the perspective of observation looked around the classroom freely, click the hot zone link, and wander in the virtual environment. Through the immersive experience of the virtual classroom, to understand the virtual classroom, to have a comparison of the real classroom felt the wonderful journey brought by the virtual world.

\subsection{Panoramic Conversion of Immersive Virtual Reality} Classroom. Finally, the whole virtual classroom scene was transformed into "panoramic mode." In order to render a 3D scene into a panorama, it needed to use the V-Ray rendering engine of 3ds Max plug-in. First, create a free camera in the virtual classroom scene and place it in the appropriate position of the scene. Select Render from the menu bar, click "Render Settings", change the output file size to 1000 width and 500 height in "Public", make sure the output image aspect ratio was $2: 1$, select the file save path and image format in the Render output. In "V-Ray", change the camera type to "spherical", check "Cover field", the Angle was $360^{\circ}$, click "Render" to output the panorama. Figure 5 shows the exported panorama. Import the rendered panoramic image into Pano2VR software and set the skin as simple. GGSK, select the output file format as HTML5, select the file output path, and click OK to generate dynamic panoramic image. You could view the panoramic VR effect of the virtual classroom through the web page version. In this way, the scene could be transformed into a VR panoramic effect, and you could use the mouse, keyboard, up, down, left, and right buttons or the hot button at the bottom of the web page to roam the virtual classroom in the "First Life perspective". You can also click the hot button to link to external resources for browsing. At this point, the entire construction process of an immersive virtual reality classroom was complete.

The constructed immersive virtual reality classroom exports a panorama through a V-Ray renderer. In Pano2VR, the panoramic pictures were converted to HTML5 format, and the web version was browsed. Students could choose their own observation angle and experience the strong visual impact brought by the virtual world through $360^{\circ}$ rotation, 


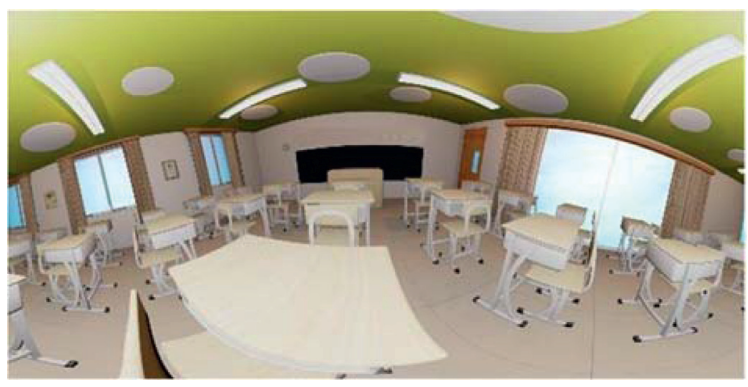

FIGURE 5: Panorama of the virtual classroom.

movement, zooming in, zooming out, and hot zone link, and felt themselves in it. The realistic, immersive virtual reality classroom allowed students to immerse themselves in it quickly. Classroom teaching in the state of immersion could improve students' interest in learning, promote students to explore and acquire knowledge actively, and help to maximize the learning effect.

4.7. Interview Data Analysis on the Application of Immersive Virtual Reality in Classroom Teaching. An immersive virtual reality classroom only provides a virtual space for students' learning or classroom teaching. A complete classroom needs to construct rich objects such as teaching AIDS, experimental equipment, and physical models in line with the curriculum content with the help of immersive virtual reality to fully present a complete classroom teaching. With the immersive, interactive, and visionary features of VIRTUAL reality, could the introduction of immersive virtual reality into the classroom really promote more effective teaching? Which courses are suitable for, and what difficulties will exist in the process of using them. Based on such doubts, through the form of interview, the author deeply discusses some attitudes, viewpoints, and views of the application of immersive virtual reality in classroom teaching and interviews five teachers, respectively. Before this, five teachers were briefly introduced to the knowledge related to immersive virtual reality, and five questions were designed, namely: Through what ways to learn about immersive virtual reality, what types of courses immersive virtual reality was suitable for, what advantages it had in teaching, what difficulties exist in the process of using it and what needs it had for future immersive virtual reality teaching, relevant data would be collected through their answers. VR in Table 1 refers to immersive virtual reality. Table 1 shows the collation of interview data results.

From the answers of the five teachers interviewed above, it could be seen that immersive virtual reality was widely used in education. All legal courses had their applications, providing an infinite virtual experience for students. Immersive virtual reality presents three-dimensional physical models. In order to verify experimental hypotheses, students could conduct simulation operations in the virtual reality space. Constantly explore and discover new knowledge and deepen the impression of knowledge so as to accelerate and consolidate the process of learning knowledge.

There were still many difficulties in the application of immersive virtual reality in teaching. Creating a scene was a big project that required a lot of effort and time. Complex model construction and interaction Settings require expertise. For an ordinary subject teacher, it was still very difficult to construct an appropriate virtual teaching scene by mastering multiple software. If the created virtual scene could not be widely used, it would also cause a huge waste of resources. Immersive virtual reality mainly provides users with a virtual space for scene experience and game entertainment, but it was still very rare to apply it to classroom teaching. If teachers only knew the basic functions and knowledge of immersive virtual reality and did not know how to integrate immersive virtual reality into the content of the course, the use value of immersive virtual reality would also be reduced. Schools, governments or other education departments had no relevant policy support and laws and regulations, making it difficult for immersive virtual reality to be introduced into the classroom.

According to the data of the questionnaire and interview, both students and teachers were optimistic about the prospect of an immersive virtual reality classroom. However, immersive virtual reality was still under development in terms of technology, hardware, and content. From the perspective of its functions, immersive virtual reality provides teachers and students with a realistic virtual reality classroom, as long as a set of virtual reality equipment can enter the exciting virtual reality world. Teachers and students through the helmet position tracking, intelligent speech recognition, gesture motion capture, close to the real natural interaction, and virtual objects interact in the real world, and at the same time in the virtual world, peers and teachers as "face to face" communication made teachers and students get the feel of real class participation that was exactly the same. At the same time, it also avoided 
TABLE 1: Teacher interview form.

\begin{tabular}{|c|c|c|c|c|}
\hline Teach & $\begin{array}{l}\text { Q1 understanding VR } \\
\text { approach }\end{array}$ & $\begin{array}{l}\text { Q2 VR applied law } \\
\text { courses }\end{array}$ & $\begin{array}{l}\text { Q1VR advantages of } \\
\text { application }\end{array}$ & $\begin{array}{c}\text { Q4 difficulties in VR application in } \\
\text { curriculum }\end{array}$ \\
\hline 1 & $\begin{array}{c}\text { News media, newspapers, } \\
\text { and magazines }\end{array}$ & Intellectual property law & $\begin{array}{c}\text { Provide a three-dimensional } \\
\text { physical model }\end{array}$ & Resource development costs are high \\
\hline 2 & $\begin{array}{l}\text { Film and television works } \\
\text { friends recommended }\end{array}$ & Administrative law & $\begin{array}{l}\text { Save the cost of equipment } \\
\text { purchase }\end{array}$ & $\begin{array}{l}\text { The model is complex and difficult to } \\
\text { construct }\end{array}$ \\
\hline 3 & $\begin{array}{l}\text { Venue experience, portal } \\
\text { website, advertising }\end{array}$ & History of legal thought & $\begin{array}{l}\text { Restore the thought scene, } \\
\text { increase the sense of presence }\end{array}$ & $\begin{array}{l}\text { The experience is not strong, and the } \\
\text { hardware and software are not mature }\end{array}$ \\
\hline 4 & $\begin{array}{c}\text { Media campaigns, search } \\
\text { engines }\end{array}$ & $\begin{array}{l}\text { Jurisprudence of } \\
\text { administrative } \\
\text { procedure }\end{array}$ & $\begin{array}{c}\text { In class, simulate cases and } \\
\text { play roles to arouse students' } \\
\text { interest }\end{array}$ & $\begin{array}{l}\text { Lack of monitoring, unable to record the } \\
\text { actual situation of students }\end{array}$ \\
\hline 5 & $\begin{array}{l}\text { TV news, website } \\
\text { recommendations }\end{array}$ & Legal logic & $\begin{array}{l}\text { Draw a 3D logic diagram to } \\
\text { enhance the logic experience }\end{array}$ & $\begin{array}{l}\text { Difficult to talk about realistic scenes } \\
\text { without notes, which is not conducive to } \\
\text { summary and induction }\end{array}$ \\
\hline
\end{tabular}

interference with external information, improved the attention of classroom teaching, and got unprecedented ultimate experience.

\section{Conclusion}

This paper proposes a divide-and-conquer partition mapping method, which can map a large scene into a small real space. Block mapping generates high-quality mapping, and AR technology is used to realize "immersive" teaching situation simulation of law course, and the following conclusions are drawn:

(1) A new representation is used for mapping, and a new formula is used for boundary constraints and distortion energy. The optimization problem is easy to solve and better optimization results are obtained.

(2) The divide-and-conquer strategy transforms global optimization into local optimization, which reduces the difficulty of optimization and is more suitable for the mapping of large scenes.

(3) Use 3ds Max software to build the virtual reality classroom of law and import the panorama rendering into Pano2VR to convert the whole scene into panorama mode. The key points of software applications are also emphasized.

(4) The application value of the constructed immersive virtual legal reality classroom in education is studied.

(5) Guide the constructed immersive virtual reality classroom panorama into HTML5 format, collect data, and analyze the questionnaire through teachers' experience and filling in the questionnaire. Understand the experience of immersive VR classrooms and the degree of acceptance of immersive VR classrooms. Deeply understand teachers' opinions on immersive virtual reality simulation and analyze the results.

\section{Data Availability}

The dataset can be accessed upon request.

\section{Conflicts of Interest}

The author declares that they have no conflicts of interest.

\section{References}

[1] N. Vaughan, V. N. Dubey, T. W. Wainwright, and R. G. Middleton, "A review of virtual reality based training simulators for orthopaedic surgery," Medical Engineering \& Physics, vol. 38, no. 2, pp. 59-71, 2016.

[2] J. L. McGrath, J. M. Taekman, P. Dev et al., "Using virtual reality simulation environments to assess competence for emergency medicine learners," Academic Emergency Medicine, vol. 25, no. 2, pp. 186-195, 2018.

[3] L. L. Wang and H. N. Cheng, "Physical education image analysis based on virtual crowd simulation and FPGA," Microprocessors and Microsystems, vol. 79, Article ID 103319, 2020.

[4] S. L. Olivares Olivares, M. V. López Cabrera, and J. E. ValdezGarcía, "Aprendizaje basado en retos: una experiencia de innovación para enfrentar problemas de salud pública," Educación Médica, vol. 19, no. 3, pp. 230-237, 2018.

[5] G. S. Letterie, "How virtual reality may enhance training in obstetrics and gynecology," Am Obstet Gynecol, vol. 187, no. 3 suppl, pp. 37-40, 2002.

[6] L. Tang, C. Zhu, and H. Luo, "5G-oriented IoT coverage enhancement and physical education resource management," Microprocessors and Microsystems, vol. 80, Article ID 103346, 2020.

[7] T. Miki, T. Iwai, K. Kotani, J. Dang, H. Sawada, and M. Miyake, "Development of a virtual reality training system for endoscope-assisted submandibular gland removal," Journal of Cranio-Maxillofacial Surgery, vol. 44, no. 11, pp. 1800-1805, 2016.

[8] H. A. Davis, C. DiStefano, and P. A. Schutz, "Identifying patterns of appraising tests in first-year college students: implications for anxiety and emotion regulation during test taking," Journal of Educational Psychology, vol. 100, no. 4, pp. 942-960, 2008.

[9] G. X. Zhong and X. Z. Zhang, "Construction of a general intelligent learning environment model," Computer Science, vol. 1, pp. 170-171+197, 2007.

[10] R. H. Huang, J. F. Yang, and Y. B. Hu, "From digital learning environment to Intelligent learning environment-The change and trend of learning environment," Open Education Research, vol. 18, no. 1, pp. 75-84, 2012. 
[11] F. H. Nie, X. L. Zhong, and S. Q. Song, "Smart classroom: conceptual features, system models and construction cases," Modern Educational Technology, vol. 23, no. 7, pp. 5-8, 2013.

[12] G.-J. Hwang, "Definition, framework and research issues of smart learning environments - a context-aware ubiquitous learning perspective," Smart Learning Environments, vol. 1, no. 1, p. 4, 2014.

[13] B. H. Shi, "Architecture design and implementation of Intelligent classroom in Internet + contemporary universities," Journal of Central China Normal University (Natural Science edition), vol. 5, no. S1, pp. 91-95, 2017.

[14] L. X. Pan, W. B. Xu, and S. B. Li, "Construction of a research and discussion type wisdom classroom based on voiceprint recognition," Experimental Technology and Management, vol. 35, no. 07, pp. 245-250, 2018.

[15] Y. Zhang, Q. Hao, and B. L. Chen, "A study on college students' classroom learning engagement and its influencing factors under smart classroom environment-a case study of "Educational Technology Research Methods course"," China Educational Technology, vol. 1, pp. 106-115, 2019.

[16] S. Kim, S.-M. Song, and Y.-I. Yoon, "Smart learning services based on smart cloud computing," Sensors, vol. 11, no. 8, pp. 7835-7850, 2011.

[17] L. Wu and C. K. Looi, "Agent prompts: scaffolding for productive reflection in an intelligent learning environment," Journal of Educational Technology \& Society, vol. 15, no. 1, pp. 339-353, 2012.

[18] M. Cheng and M. X. Shan, "A review of research on intelligent learning environment," Modern Educational Technology, vol. 23, pp. 25-28, 2013.

[19] P. Temdee, "Ubiquitous learning environment: smart learning platform with multi-agent architecture," Wireless Personal Communications, vol. 76, no. 3, pp. 627-641, 2014.

[20] D. Griol, J. M. Molina, and Z. Callejas, "An approach to develop intelligent learning environments by means of immersive virtual worlds," Journal of Ambient Intelligence and Smart Environments, vol. 6, no. 2, pp. 237-255, 2014.

[21] Kinshuk, N. S. Chen, N.-S. Chen, I.-L. Cheng, and S. W. Chew, "Evolution is not enough: revolutionizing current learning environments to smart learning environments," International Journal of Artificial Intelligence in Education, vol. 26, no. 2, pp. 561-581, 2016.

[22] N. T. Le and N. Pinkwart, "A Smart Problem Solving environment," 2019, https://cses.informatik. 